\title{
Active Comparator Trial Designs Used to Promote Development of Innovative New Medications
}

John S. Barbieri, MD, MBA; Jerry K.L. Tan, MD, FRCPC; Adewole S. Adamson, MD, MPP

\section{PRACTICE POINTS}

- When evaluating a new treatment, it is important to consider not only whether it is effective but also whether it provides additional value compared to existing treatment options.

- Encouraging active comparator trials will provide clinicians and patients with important data to guide decision-making regarding the most appropriate treatment options. $\bigcup^{p}$ pending on medications is expected to grow from $\$ 344$ billion in 2018 to $\$ 420$ billion in 2023, largely driven by the introduction of new branded drugs. ${ }^{1}$ These costs place substantial financial burden on patients, with nearly $30 \%$ of patients not taking their prescriptions as directed because of costs. Although many new medications have transformed how we care for patients, others may not offer meaningful benefit over existing less-costly alternatives that are supported by declining effect sizes of conventional placebo-controlled trials. ${ }^{2}$ Most medications are approved based on placebo-controlled trial data that does not include an arm comparing the new drug to standard of care, leaving clinicians and patients unable to make meaningful comparisons when deciding on the most appropriate or cost-effective treatment. We consider ways in which clinicians, patients, payers, and regulators could compel more meaningful trials from industry.

Although we often look to the US Food and Drug Administration (FDA) to ensure rigorous and appropriate testing of new medications, the primary mission of the FDA is to ensure efficacy and safety. As a result, pharmaceutical companies seeking approval in the United States have little incentive to go beyond providing the minimal level of evidence required: placebo-controlled randomized trials. Although these trials provide important data on whether a treatment works and its associated risks, they do not provide data on comparative effectiveness. When relevant inexpensive medications are already on the market for the same indication, these placebocontrolled trials provide inadequate evidence to guide clinical decision-making. This issue is particularly relevant in dermatology given how easily topical medications can be combined or reformulated to pursue additional market exclusivity. The addition of an active comparator arm represents an important opportunity to improve the value of these studies.

In the pivotal trials of clindamycin phosphate $1.2 \%$ benzoyl peroxide $2.5 \%$ gel for the treatment of acne, the experimental group was not only compared to vehicle but also the active comparator arms of clindamycin alone and benzoyl peroxide alone. The mean percentage change in total lesions was $47.9 \%$ with clindamycin phosphate $1.2 \%$-benzoyl peroxide $2.5 \%$ gel, $41.6 \%$ with the active

Dr. Barbieri is from the Department of Dermatology, Perelman School of Medicine, University of Pennsylvania, Philadelphia. Dr. Tan is from the Schulich School of Medicine and Dentistry, Western University, Windsor, Ontario, Canada. Dr. Adamson is from the Division of Dermatology, Department of Internal Medicine, University of Texas at Austin.

The authors report no conflict of interest.

Correspondence: John S. Barbieri, MD, MBA, Perelman Center for Advanced Medicine, 7 S Pavilion, 3400 Civic Center Blvd, Philadelphia, PA 19104 (john.barbieri@uphs.upenn.edu).

doi:10.12788/cutis.0067 
comparator arm of benzoyl peroxide alone, $40.4 \%$ with the active comparator arm of clindamycin alone, and $26.2 \%$ for vehicle. ${ }^{3}$ With these data in mind, clinicians and patients can decide whether the additional benefit of this new product over benzoyl peroxide alone is worth the increased cost.

In contrast, the trials of dapsone gel $7.5 \%$ for the treatment of acne did not include an active comparator. The mean percentage change in total lesions was $48.9 \%$ for dapsone gel and $43.2 \%$ for vehicle. ${ }^{4}$ Given these data, it is possible that dapsone gel may be no more effective, or possibly less effective, than alternatives such as benzoyl peroxide or other topical antibiotics. Nevertheless, dapsone annual sales were more than $\$ 200$ million in 2016, ${ }^{5}$ suggesting that effectively marketed new products can achieve high sales even without convincing evidence of their value compared to standard of care. Although dapsone may be a useful treatment, we cannot effectively make patient-centered clinical decisions given the lack of an active comparator trial design.

This issue is not limited to acne. Phase 3 trials of halobetasol propionate foam $0.05 \%$ for psoriasis and crisaborole for atopic dermatitis also did not include an active comparator arm. ${ }^{6,7}$ Given that topical steroids—and calcineurin inhibitors for atopic dermatitis-are mainstays of treatment for each condition, it is difficult to determine whether these new treatments offer meaningful advantages over existing options and how to incorporate them into our management strategies.

Unfortunately, expensive new medications that are adopted without convincing evidence of their benefit above standard of care can put patients at risk for financial toxicity, either directly through higher out-of-pocket costs or indirectly through higher premiums. Given the impact of rising medication costs on clinicians, patients, and payers, we propose several approaches these stakeholders could adopt to encourage the use of active comparator trial designs.

Clinicians and patients can encourage these trials by remaining skeptical of new treatments that were only compared to vehicle or placebo. Because new medications often are more expensive, clinicians and patients could avoid using these treatments without evidence of either increased efficacy or improved safety and tolerability. In addition, health care institutions should consider reducing pharmaceutical representatives' access to clinicians to encourage treatment decisions based on the published literature and comparative effectiveness data rather than marketing.

Payers, such as Medicare, also could play a role by requiring active comparator trials for coverage of new medications, particularly when there are already other effective treatments available or other medications in the same class. Payers also could give preferred coverage tier or step therapy status to medications that demonstrate value over existing options.
Although regulatory approaches to increase use of active comparator designs may be more politically challenging to introduce, these options would be more administratively robust. The FDA or a novel regulatory body could require that new treatments demonstrate value in addition to safety and efficacy. This approach would be similar to the role of The National Institute for Health and Care Excellence in the United Kingdom or the recommendations of the European Medicines Agency. Such a group also could provide independent adjudication to ensure appropriate selection of a relevant active comparator. Another approach would be to give extended market exclusivity to medications that are approved based on trials including an additional active comparator arm, an approach used by the European Medicines Agency.

Any approach that encourages increased use of active comparator trials is not without potential downsides. It will be important to avoid unintended consequences of reduced research for rare diseases with smaller markets that may not be able to support the increased cost of these trials. As a result, it would be reasonable to forgo active comparator designs for mediations indicated for rare and orphan diseases or for medications with novel mechanisms of action.

Another argument against including an active comparator arm is that it may stifle innovation by driving up the cost of conducting trials; however, if a product is so marginally innovative that it cannot demonstrate superior safety or efficacy to an existing product, such a new treatment may not be worth the increased cost. In addition, patients provide a notable contribution by participating in these trials, and it is important to ensure that their efforts result in the highest-quality data possible. Furthermore, given the adverse physical and psychosocial impact of a wide variety of dermatologic diseases, the inclusion of an active comparator arm reduces the likelihood that patients will receive placebo, which will make these trials more ethical when effective treatments are available. ${ }^{8}$ By raising the bar, we can encourage pharmaceutical companies to pursue novel approaches that are more likely to have a revolutionary impact rather than minor modifications or formulations that offer little to no benefit at substantially increased cost.

Although some recent clinical trials in dermatology have included active comparators, many new medications continue to be introduced without any evidence of how they compare to existing standards of care. Until clinicians, patients, payers, and regulators demand that pharmaceutical companies conduct the necessary trials to not only demonstrate whether a treatment is effective and safe but also how it provides value, there will be continued introduction of marginal innovations rather than revolutionary treatments that improve patients' lives. The next time a new medication is approved, as clinicians, patients, and payers, we must ask ourselves, is this treatment worth it? 


\section{REFERENCES}

1. Aitken M, Kleinrock M. Medicine Use and Spending in the U.S.: A Review of 2018 and Outlook to 2023. IQVIA Institute for Human Data Science. https://www.iqvia.com/insights/the -iqvia-institute/reports/medicine-use-and-spending-in-the-us-a -review-of-2018-and-outlook-to-2023. Published May 9, 2019. Accessed August 15, 2020.

2. Olfson M, Marcus SC. Decline in placebo-controlled trial results suggests new directions for comparative effectiveness research. Health Aff Proj Hope. 2019;32:1116-1125.

3. Thiboutot $\mathrm{D}$, Zaenglein $\mathrm{A}$, Weiss J, et al. An aqueous gel fixed combination of clindamycin phosphate $1.2 \%$ and benzoyl peroxide $2.5 \%$ for the once-daily treatment of moderate to severe acne vulgaris: assessment of efficacy and safety in 2813 patients. J Am Acad Dermatol. 2008;59:792-800.

4. Eichenfield LF, Lain T, Frankel EH, et al. Efficacy and safety of once-daily dapsone gel, $7.5 \%$ for treatment of adolescents and adults with acne vulgaris: second of two identically designed, large, multicenter, randomized, vehicle-controlled trials. J Drugs Dermatol. 2016;15:962-969.

5. Allergan. 2017 Form 10-K. https://www.abbvie.com/content /dam/abbvie-dotcom/uploads/PDFs/allergan/allergan-annual-report -form-10K-123117.pdf. Accessed August 19, 2020.

6. Paller AS, Tom WL, Lebwohl MG, et al. Efficacy and safety of crisaborole ointment, a novel, nonsteroidal phosphodiesterase 4 (PDE4) inhibitor for the topical treatment of atopic dermatitis (AD) in children and adults. J Am Acad Dermatol. 2016;75:494-503.e6.

7. Bhatia N, Stein Gold L, Kircik LH, et al. Two multicenter, randomized, double-blind, parallel group comparison studies of a novel foam formulation of halobetasol propionate, $0.05 \%$ vs its vehicle in adult subjects with plaque psoriasis. J Drugs Dermatol. 2019;18:790-796.

8. Temple R, Ellenberg SS. Placebo-controlled trials and active-control trials in the evaluation of new treatments. part 1: ethical and scientific issues. Ann Intern Med. 2000;133:455-463. 